\title{
Unscented Particle Filter for SOC Estimation Algorithm Based on a Dynamic Parameter Identification
}

\author{
Fang Liu $\mathbb{D}^{1,2}$ Jie Ma $\mathbb{D}^{1},{ }^{1}$ and Weixing Su${ }^{1}$ \\ ${ }^{1}$ School of Computer Science and Technology, Tianjin Polytechnic University, Tianjin 300387, China \\ ${ }^{2}$ Tianjin Qingyuan Electric Vehicle Limited Liability Company, Tianjin 300457, China \\ Correspondence should be addressed to Fang Liu; liufang19830311@163.com and Jie Ma; 573339374@qq.com
}

Received 26 January 2019; Revised 23 March 2019; Accepted 14 April 2019; Published 22 April 2019

Academic Editor: Zhiwei Gao

Copyright (c) 2019 Fang Liu et al. This is an open access article distributed under the Creative Commons Attribution License, which permits unrestricted use, distribution, and reproduction in any medium, provided the original work is properly cited.

\begin{abstract}
In order to solve the problem that the model-based State of Charge (SOC) estimation method is too dependent on the model parameters in the SOC estimation of electric vehicles, an improved genetic algorithm is proposed in this paper. The method has the advantages of being able to quickly determine the search range, reducing the probability of falling into local optimum, and having high recognition accuracy. Then we can realize online dynamic identification of power battery model parameters and improve the accuracy of model parameter identification. In addition, considering the complex application environment and operating conditions of electric vehicles, an SOC estimation method based on improved genetic algorithm and unscented particle filter (improved GA-UPF) is proposed. And we compare the improved GA-UPF algorithm with the least square unscented particle filter (LS-UPF) and improved GA unscented Kalman filter (improved GA-UKF) algorithm. The comparison results show that the improved GA-UPF algorithm proposed in this paper has higher estimation accuracy and better stability. It also reflects the practicability and accuracy of the improved GA parameter identification algorithm proposed in this paper.
\end{abstract}

\section{Introduction}

Power battery is one of the core components of electric vehicles. Accurate estimation and management of its key state is the core issue of electric vehicle development. State of charge (SOC) is one of the key states, which has a great significance for energy-optimized management and healthy life management of electric vehicles. Accurate estimation of SOC can maximize the utilization rate of power battery, extend battery life, and ultimately reduce power battery cost.

In recent years, a series of online estimation methods of battery SOC are proposed for the SOC estimation of electric vehicles [1-27], including the ampere hour integral method [5-7], the neural network method [19-22], the filter method based on the battery model [8-13], and so on. The calculation of the ampere-time integral method is small and it is easy to implement, but its estimation error is large because of the accumulated error. The neural network method is easy to build an intelligent model, but it requires a large amount of experimental data to train the neural network model, and the amount of calculation is large. In order to overcome the effects of cumulative errors and excessive computational problems, a series of the filter method based on the battery model have been proposed [14-18]. Piller et al. [8] proposed the Kalman filter (KF) method for SOC estimation, which has closed-loop correction structure. However, the KF is a linear filter; the battery model is a nonlinear model; it will produce a large error. In order to get better results, scholars proposed a lot of methods based on the extended Kalman filter (EKF) $[10,13-15,26]$ and the unscented Kalman filter (UKF) [16-18, 24]. Wang et al. [26] proposed a dual extended Kalman filter method for the SOC estimation. Plett $[14,15,18]$ proposed EKF and UKF for SOC and battery model parameter estimation. Wang [17] proposed an improved unscented Kalman filter for the SOC estimation. However, the accuracy of EKF and UKF both are not sufficiently enough to be used in engineering applications. Their accuracy is very dependent on the accuracy of the battery model parameters. Additionally, their noise distribution must be the Gaussian distribution, which is more deviating from the real noise environment. 
According the above analysis of mainstream SOC estimation methods, the special background of power battery application in electric vehicle field, and its engineering requirements, this paper proposes an SOC estimation method based on improved GA-UPF algorithm. The algorithm innovations and advantages are as follows.

(1) We propose an optimization strategy based on least squares method to initialize GA initial population. This strategy is used to make the GA algorithm quickly and accurately converge and reduce the computational complexity of GA algorithm and its probability of falling into local optimum.

(2) We propose an improved GA algorithm that is more suitable for nonlinear complex system identification of the power battery model. The algorithm can reduce the complexity of the algorithm and obtain more accurate model parameters. Finally, the algorithm can improve the estimation accuracy of SOC.

(3) We analyze the accuracy, complexity, dynamic characteristics, and other factors of many power battery models, such as the Rint model, the Thevenin model, the Partnership for a New Generation of Vehicles (PNGV) model [23, 25], the General nonlinear (GNL) model, and so on. Then we choose the PNGV model, which is more able to characterize the internal changes in the battery charging and discharging process and has practical physical significance.

(4) We consider the measurement data of the power battery with complex noise, abnormal points, and severe fluctuations, and then use the unscented particle filter (UPF) algorithm for SOC estimation. The advantages of UPF are that it has strong robustness, computational accuracy, and better suppression effect on colored noise, which is more in line with actual engineering requirements.

The rest of paper is organized as follows. In Section 2, we introduce the power battery model and the parameter identification method based on the improved GA algorithm. In Section 3, we introduce an SOC estimation algorithm based on improved GA-UPF. In Section 4, simulations and comparison tests based on the PNGV model are presented to verify the superiority of the proposed algorithm. In Section 5, the improved GA-UPF algorithm proposed in this paper is summarized.

\section{Power Battery Equivalent Model and Model Parameter Identification}

2.1. PNGV Model. The PNGV model was proposed for the 2001 US New Generation Vehicle Cooperation Program to model power batteries in the automotive field [23]. The PNGV model has the advantages of low processor requirements, easy implementation, and being suitable for describing the dynamic characteristics of the battery. It is beneficial to the analysis of battery characteristics and is more suitable for the modeling of lithium ion batteries. Therefore, considering the complexity and accuracy of the model, the PNGV model is adopted and displayed. The model structure is shown in Figure 1.

In the figure, $U_{o c v}$ is the open circuit voltage, $C_{b}$ is the cell capacitance, $R_{e}$ is the cell internal resistance, $R_{p}$ is the cell polarized resistance, $C_{p}$ is the cell polarized capacitance, $U_{d}$ is the cell terminal voltage, $I_{t}$ is the circuit current, $U_{b}$ is the terminal voltage across the capacitance $C_{b}$, and $U_{p}$ is the terminal voltage across the capacitance $C_{p}$.

According to the PNGV model shown in Figure 1, the measurement equation for the PNGV battery model can be expressed by

$$
\begin{aligned}
& U_{d}=U_{o c v}(S O C, \mathrm{~T})-U_{\mathrm{b}}-U_{p}-I \times \mathrm{R}_{\mathrm{e}} \\
& \dot{U}_{b}=\frac{1}{C_{b}} \times I \\
& \dot{U}_{p}=-\frac{1}{C_{p} \times R_{p}} \times U_{p}+\frac{1}{C_{p}} \times I
\end{aligned}
$$

where $U_{o c v}(S O C, T)$ is the open circuit voltage at different SOC values and different temperatures, $\dot{U}_{b}$ is the derivation operation of the voltage $U_{b}$, and $\dot{U}_{p}$ is the derivation operation of the voltage $U_{p}$.

Then we discrete (1), it can be expressed by

$$
\begin{aligned}
U_{d}^{t}= & U_{o c v}\left(S O C_{t}, T_{t}\right)-U_{b}^{t}-U_{p}^{t}-I_{t} \times R_{\mathrm{e}} \\
U_{b}^{t}= & U_{b}^{t-1}+\left(\frac{\Delta t}{C_{b}}\right) I_{t} \\
U_{p}^{t}= & \exp \left(\frac{-\Delta t}{\left(C_{p} R_{p}\right)}\right) U_{p}^{t-1} \\
& +R_{p}\left(1-\exp \left(\frac{-\Delta t}{\left(C_{p} R_{p}\right)}\right)\right) I_{t}
\end{aligned}
$$

where $U_{d}^{t}$ is the terminal voltage at time $t, U_{o c v}\left(S O C_{t}, T_{t}\right)$ is the open circuit voltage related to $S O C_{t}$ and the temperature value at time $t, U_{b}^{t}$ and $U_{p}^{t}$ are the terminal voltages across $C_{b}$ and $C_{p}$ in the PNGV model at time $t, \Delta t$ is the simple period, $I_{t}$ is the measured current of the power battery at the time $t$, and Para $=\left[C_{b}, R_{p}, C_{p}, R_{e}\right]$ is the parameter vector to be identified in the PNGV model.

2.2. Battery Model Parameters Identification Based on Improved GA Algorithm. The traditional GA algorithm performs parameter identification by searching globally to find the optimal solution and does not require difficult mathematical model expressions [28]. Therefore, it is suitable for the parameter identification of nonstationary process characterization models, such as electric vehicle power battery model, which has nonlinear characteristics, time-varying parameters, complex environmental noise, sharp fluctuations in collected data, and more transient spikes. However, it has a long search time and fall into local optimum easily because of the characteristics of its global search. Aiming at the problem, an improved GA search strategy is proposed. In this strategy, 


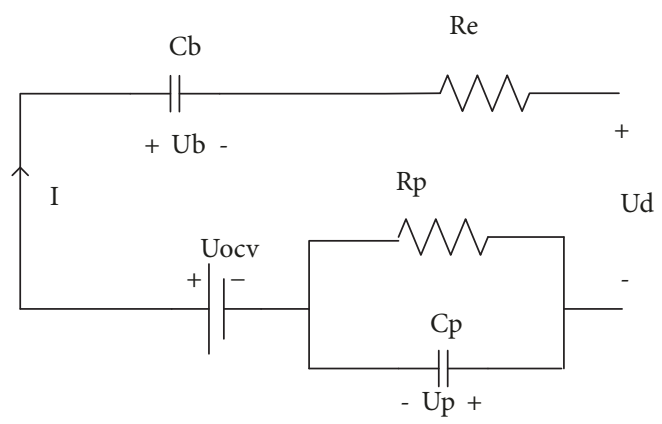

FIGURE 1: PNGV battery model.

we use the least squares method to initialize the search range at the initial moment of the algorithm. It can effectively shorten the search time, avoid falling into local optimum, and reduce the complexity of the algorithm. The flow chart of improved GA algorithm is shown in Figure 2, where $g$ is the current iterations of the improved GA algorithm and $G$ is the maximum number of iterations of the improved GA algorithm. The improved GA algorithm is calculated as follows.

Step 1. Search range initialization based on LS algorithm [25]. We can obtain (3) by transforming (2):

$$
U_{d}^{t}=a \times U_{d}^{t-1}+b \times U_{d}^{t-2}+c \times I_{t}+d \times I_{t-1}+e \times I_{t-2}
$$

where $U_{d}^{t-1}, U_{d}^{t-2}, I_{t}, I_{t-1}, I_{t-2}$ are measurable values. Based on (3), we can get the vector $\gamma$ expression by LS algorithm. Equation (4) is as follows:

$$
\begin{aligned}
& \gamma=\left[\begin{array}{l}
a \\
b \\
c \\
d \\
e
\end{array}\right] \\
& =\left[\begin{array}{c}
1+\exp (-\theta) \\
-\exp (-\theta) \\
-R_{e} \\
(1+\exp (-\theta)) R_{e}-(1-\exp (-\theta)) R_{p}-\frac{1}{C_{p}} \\
(1-\exp (-\theta)) R_{p}-\exp (-\theta) R_{e}+\frac{\exp (-\theta)}{C_{b}}
\end{array}\right]
\end{aligned}
$$

where $\theta=\Delta t /\left(C_{p} R_{p}\right)$.
The expression of Para $=\left[C_{b}, R_{p}, C_{p}, R_{e}\right]$ obtained by (4) is as follows:

$$
\operatorname{Para}=\left[\begin{array}{c}
C_{b} \\
R_{p} \\
C_{p} \\
R_{e}
\end{array}\right]^{T}=\left[\begin{array}{c}
-\frac{1+b}{c+d+e} \\
\frac{e-b \times d+c \times b^{2}}{(1+\mathrm{b})^{2}} \\
-\frac{\Delta t}{c \times \ln (-\mathrm{b})} \\
-c
\end{array}\right]^{T}
$$

Then, we can use the voltage values and the current values measured at the previous $\mathrm{L}$ ( $\mathrm{L}$ is the time window of the least squares algorithm) time to obtain the vector $\gamma=$ $[\mathrm{a}, \mathrm{b}, \mathrm{c}, \mathrm{d}, \mathrm{e}]^{T}$. The equation is as follows:

$$
\gamma=\left(\varphi_{t}^{T} \cdot \varphi_{t}\right)^{-1} \cdot \varphi_{t}^{T} \cdot U_{d}^{t}
$$

where $\varphi_{t}=\left[U_{d}^{t-1}, U_{d}^{t-2}, I_{t}, I_{t-1}, I_{t-2}\right]$.

According to (5), Para $_{t}$ can be calculated as the initial parameter value of the PNGV model. Then, we use Para ${ }_{t}$ as the center point and $r$ as the radius in the high-dimensional space (the spatial dimension is equal to the dimension of the PNGV model parameters) to determine rang $_{\text {para }_{t}}$ (the initial search range) of the improved GA algorithm. Equation (7) is as follows:

$$
\begin{aligned}
\operatorname{rang}_{\text {para }_{t}} & =[\min , \text { max }] \\
& =\left[\left(\text { Para }_{t}-r\right) \pm \varepsilon,\left(\text { Para }_{t}+r\right) \pm \varepsilon\right]
\end{aligned}
$$

where $\varepsilon$ is a constant that $\varepsilon \longrightarrow 0$.

Step 2. We use a random uniform distribution to generate the initial population. The equation is as follows:

$$
\operatorname{Para}_{t}^{k}(0)=\operatorname{rand}(0,1) \times(\max -\min )+\operatorname{Para}_{t}
$$

where $k=1, \ldots, n, n$ is the number of populations, $\operatorname{Para}_{t}^{k}(0)$ is the $\mathrm{k}$-th individual value of the initial population at time $t, \operatorname{rand}(0,1)$ is the random vector. 


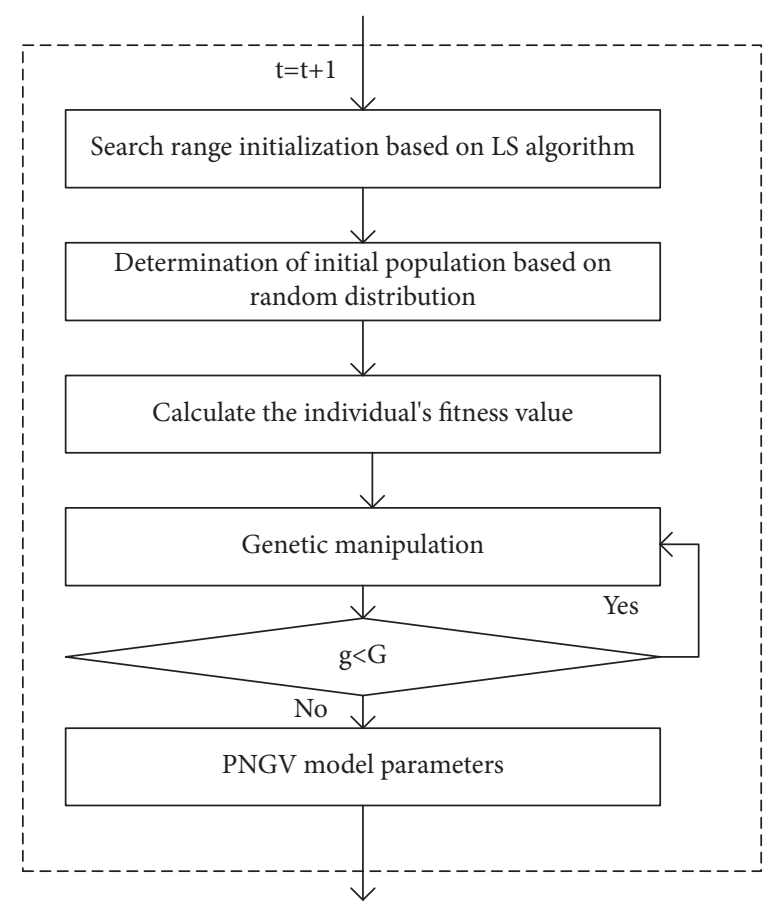

FIgURE 2: Improved GA algorithm flow chart.

Step 3. The individual's fitness value needs to be calculated. The equation for calculating individual fitness is as follows [28]:

$$
f i t_{t}^{k}(\mathrm{~g})=\left|\widehat{U}_{d}^{k}(\mathrm{~g})-U_{d}^{t}\right|
$$

where $k=1, \ldots, n, g=1, \ldots, G, G$ is the maximum number of iterations, $f_{i t}^{k}(g)$ is the fitness value of the g-th generation of the k-th individual at time t, $\widehat{U}_{d}^{k}(\mathrm{~g})$ is an estimated value of the battery terminal voltage at time $t$ of the PNGV model calculated from the $\mathrm{k}$-th individual of the g-th generation.

Step 4. Genetic manipulation is as follows:

(a) Select operation: we use the roulette strategy to select $\mathrm{N}$ individuals [28].

(b) Cross operation: we generate $r_{c o r}(k)=\operatorname{rand}(0,1)$. If $r_{c o r}(k)<P_{c o r}$, the $\mathrm{k}$-th individual is selected for cross operation. The crossover equation is as follows:

$$
\begin{aligned}
& \operatorname{Para}_{t}^{a}\left(\mathrm{~g}^{-}\right)=\rho \operatorname{Para}_{t}^{a}(\mathrm{~g})+(1-\rho) \operatorname{Para}_{t}^{b}(\mathrm{~g}) \\
& \operatorname{Para}_{t}^{b}\left(\mathrm{~g}^{-}\right)=\rho \operatorname{Para}_{t}^{b}(\mathrm{~g})+(1-\rho) \operatorname{Para}_{t}^{a}(\mathrm{~g})
\end{aligned}
$$

where $\operatorname{Para}_{t}^{a}\left(g^{-}\right)$is the a-th individual at time $t$ after the cross operation, $\operatorname{Para}_{t}^{b}\left(g^{-}\right)$is the b-th individual at time $t$ after the cross operation, $\rho$ is the random number generated between $[0,1], r_{c o r}(k)=\operatorname{rand}(0,1)$, and $P_{c o r}$ is the crossover probability.

(c) Mutation operation: We generate $r_{\text {mut }}(k) \in[0,1]$. If $r_{\text {mut }}(k)<P_{\text {mut }}$, the $\mathrm{k}$-th individual is selected for mutate operation. The equation for the mutation operation is as follows:

$$
\begin{aligned}
& \operatorname{Para}_{t}^{c}\left(\mathrm{~g}^{+}\right) \\
& = \begin{cases}\operatorname{Para}_{t}^{c}\left(\mathrm{~g}^{-}\right)+\left(\max -\operatorname{Para}_{t}^{c}\left(\mathrm{~g}^{-}\right)\right)(1-f(g)), & \mu \geq 0.5 \\
\operatorname{Para}_{t}^{c}\left(\mathrm{~g}^{+}\right)+\left(\operatorname{Para}_{t}^{c}\left(\mathrm{~g}^{-}\right)-\min \right)(1-f(g)), & \mu \geq 0.5\end{cases}
\end{aligned}
$$

where $f(g)=\mu^{\prime}(1-g / G)^{2}, \mu$ and $\mu^{\prime}$ are the random number between $[0,1], g=1,2, \ldots, G, r_{m u t}(k)=\operatorname{rand}(0,1)$, and $P_{\text {mut }}$ is the mutation probability.

\section{Power Battery SOC Estimation Algorithm Based on Improved GA-UPF}

The power battery has strong noise and complicated driving conditions in electric vehicle application. The collected battery data contains a lot of noise and abnormal points; the data changes drastically and there are many peak transient values. Thus, we want the SOC estimation method to have strong robustness, antinoise ability and self-correction capability. Based on the above considerations, the UPF algorithm is used for SOC estimation. However, the estimation accuracy of the filtering algorithm depends on the accuracy of the power battery model. Thus, we propose a power battery SOC estimation method based on the improved GA-UPF algorithm, which makes it more suitable for the estimation of the power battery SOC in the electric vehicle field. The flow chart is shown in Figure 3:

(1) Set basic parameters: several control parameters should be set, including number of particles $\mathrm{N}$, number of population $n$, maximum number of genetic iterations $G$, the 


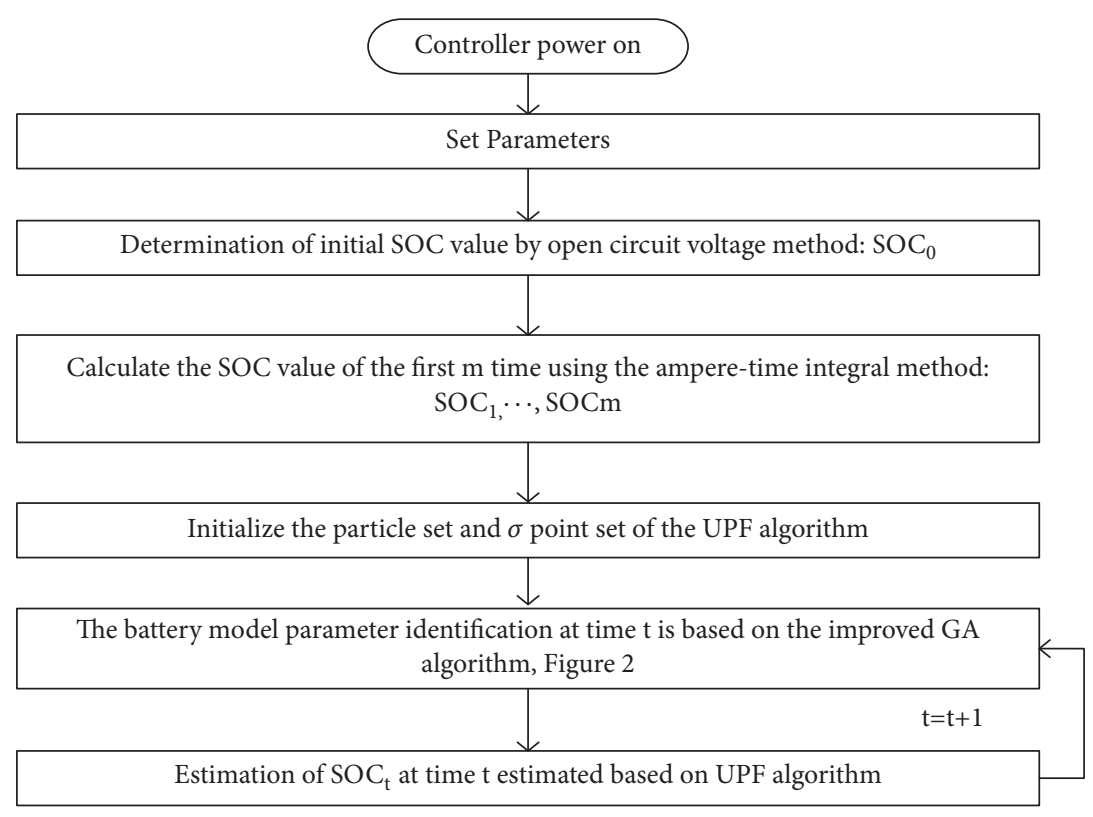

FIGURE 3: Improved GA-UPF algorithm flow chart.

crossover probability $P_{\text {cor }}$, the mutation probability $P_{m u t}, \sigma$ point search range $\alpha$, state variable scale parameter $k$, and covariance precision parameter $\beta$.

(2) Determine the initial SOC by open circuit voltage method: this part is only calculated once when the electric vehicle battery management system (BMS) is used for the first time. At this time, the BMS is powered on, but the power battery main relay is not closed and the power battery is left standing long enough. Therefore, we can believe that $U_{d}^{0}$ equals $U_{o c v}$.

(3) Calculate the SOC of the first m time: we use the ampere-time integral method:

$$
\mathrm{SOC}_{t}=\mathrm{SOC}_{t-1}+\frac{\eta\left(T_{t}, I_{t}\right) \times \Delta t}{C_{n}} \times I_{t}
$$

where $t$ is the time, $t=1, \ldots, m, m \ll T_{S O H}, T_{S O H}$ is the time when the power battery is terminated, $\eta\left(T_{t}, I_{t}\right)$ is the battery capacity factor associated with $T_{t}$ and $I_{t}$ (positive at discharge and negative at charge) at time $t, C_{n}$ is rated capacity, $\Delta \mathrm{t}$ is the simple period.

(4) Initialize the particle set and $\sigma$ point set of the UPF algorithm: when $t=m$, we generate Part $_{t}=$ $\left\{\operatorname{Part}_{t}(1), \operatorname{Part}_{t}(2), \ldots, \operatorname{Part}_{t}(\mathrm{~N})\right\}$ by a random uniform distribution; each particle has a weight of $1 / N ; N$ is the number of particles.

Then we build $X_{t}=\left\{X_{t}(1), X_{t}(2), \ldots, X_{t}(N)\right\}$ for each particle; the equation is as follows:

$$
\begin{aligned}
& X_{t}(i) \\
& =\left[\begin{array}{c}
\operatorname{Part}_{t}(i) \\
\operatorname{Part}_{t}(i)+\sqrt{(1+\lambda) \mathrm{P}_{t}^{i}(\mathrm{j})}, j=1, \ldots, l \\
\operatorname{Part}_{t}(i)-\sqrt{(1+\lambda) \mathrm{P}_{t}^{i}(\mathrm{j})}, \quad j=l+1, \ldots, 2 l
\end{array}\right]
\end{aligned}
$$

where $i=1,2, \ldots, N, l$ is the dimension of the state quantity of the state equation, $\lambda$ is the scale when generating the $\sigma$ point set.

(5) Parameter identification of the PNGV model based on improved GA algorithm at time t: this part of the content has been introduced in Section 2.2.

(6) SOC estimation based on the UPF algorithm: we obtained the state-space measurement equations of the UPF algorithm based on (2) and (12). SOC $t$ is the state variable at time $t, U_{d}^{t}$ is the measurable variable at time $t$. The estimation steps are as follows.

(a) We perform UT transformation on the $\sigma$ point set generated by (13) and obtain the set of particles at time $t$ $[9,15,17]$. The equations are as follows:

$$
\begin{aligned}
& X_{t}\left(i^{-}\right)=X_{t-1}\left(i^{-}\right)+\frac{\eta\left(T_{t}, I_{t}\right) \times \Delta t}{C_{n}} \times I_{t} \\
& W_{m}^{j}= \begin{cases}\frac{\lambda}{l+\lambda}, \quad j=0 \\
\frac{1}{2(l+\lambda)}, \quad j \neq 0\end{cases} \\
& W_{c}^{j}= \begin{cases}\frac{\lambda}{l+\lambda}+\left(1+\alpha^{2}-\beta\right), & j=0 \\
\frac{1}{2(l+\lambda)}, & j \neq 0\end{cases} \\
& \delta_{t}\left(i^{-}\right)=\sum_{j=0}^{2 l} W_{m}^{j} X_{t}^{j}\left(i^{-}\right) \\
& P_{t}\left(i^{-}\right) \\
& \quad=\sum_{j=0}^{2 l} W_{m}^{j}\left(X_{t}^{j}\left(i^{-}\right)-\delta_{t}\left(i^{-}\right)\right)\left(X_{t}^{j}\left(i^{-}\right)-\delta_{t}\left(i^{-}\right)\right)^{T}
\end{aligned}
$$




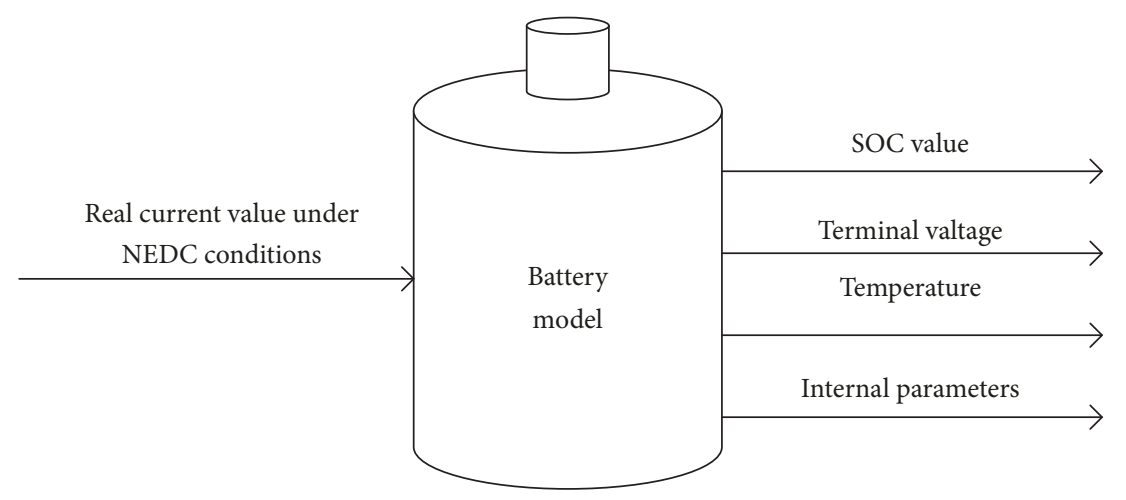

FIGURE 4: Simulation data generation process.

$$
\begin{aligned}
& \varphi_{t}\left(i^{-}\right)=\widehat{U}_{d}\left(X_{t}\left(i^{-}\right), I_{t}\right) \\
& Y_{t}\left(i^{-}\right)=\sum_{j=0}^{2 l} W_{m}^{j} \varphi_{t}^{j}\left(i^{-}\right) \\
& P_{y_{t}, y_{t}}=\sum_{j=0}^{2 l} W_{c}^{j}\left(\varphi_{t}^{j}\left(i^{-}\right)-Y_{t}\left(i^{-}\right)\right)\left(\varphi_{t}^{j}\left(i^{-}\right)-Y_{t}\left(i^{-}\right)\right)^{T} \\
& P_{x_{t}, y_{t}}=\sum_{j=0}^{2 l} W_{c}^{j}\left(X_{t}^{j}\left(i^{-}\right)-\delta_{t}\left(i^{-}\right)\right)\left(\varphi_{t}^{j}\left(i^{-}\right)-Y_{t}\left(i^{-}\right)\right)^{T} \\
& K_{t}=P_{x_{t}, y_{t}} P_{y_{t}, y_{t}}{ }^{-1} \\
& X_{t}\left(i^{+}\right)=X_{t}\left(i^{-}\right)+K_{t}\left(U_{d}^{t}-Y_{t}\left(i^{-}\right)\right)
\end{aligned}
$$

where $X_{t}\left(i^{-}\right)=\left[X_{t}^{0}\left(i^{-}\right), \ldots, X_{t}^{j}\left(i^{-}\right), \ldots, X_{t}^{2 l}\left(i^{-}\right)\right]^{T}$ is the $\sigma$ set of the $i$ th particle at time $t, W_{m}=\left[W_{m}^{0}, \ldots, W_{m}^{j}, \ldots, W_{m}^{2 l}\right]$ is the weight vector of the $\sigma$ set, $\lambda=\alpha^{2}(l+k)-l, \alpha \epsilon$ $(0,1], \beta$ is the covariance precision; we can improve variance accuracy by adjusting $\beta ; W_{c}=\left[W_{c}^{0}, \ldots, W_{c}^{j}, \ldots, W_{c}^{2 l}\right]$ is the weight vector of the covariance of the $\sigma$ set; $\varphi_{t}\left(i^{-}\right)=$ $\left[\varphi_{t}\left(i^{-}\right), \ldots, \varphi_{t}^{j}\left(i^{-}\right), \ldots, \varphi_{t}^{2 l}\left(i^{-}\right)\right]$is the model terminal voltage vector according to $\sigma$ set.

(b) We calculate the weight values for each particle and normalize them. The equation is as follows:

$$
\begin{aligned}
& q_{i}=\frac{1}{\sqrt{2 \pi R}} \exp \left(\frac{-\left(U_{d}^{t}-\widehat{U}_{d}\left(X_{t}\left(i^{+}\right), I_{t}\right)\right)^{2}}{2 R}\right) \\
& \bar{q}_{i}=\frac{q_{i}}{\sum_{i=1}^{N} q_{i}}
\end{aligned}
$$
follows:

(c) We calculate the SOC at time t. The equation is as

$$
\mathrm{SOC}_{t}=\sum_{i=1}^{N} X_{t}\left(\mathrm{i}^{+}\right) \times \bar{q}_{i}
$$

(d) The $\sigma$ set is updated by (13).

\section{Simulations and Experimental Results}

In order to verify the practicability of the SOC estimation method based on the improved GA-UPF algorithm, we initialize the power battery model of DSPACE with the internal parameters of a certain 18650 battery of LG and then construct the power battery simulation model used for algorithm verification. This model can accurately characterize the dynamic characteristics and thermal response characteristics of the power battery to a certain extent. In this section, we need to verify and compare the model identification accuracy of the improved GA algorithm and analyze the accuracy of the improved GA-UPF algorithm in the noise environment. Therefore, it is considered appropriate to use the battery model.

Based on the construction of the battery model, this paper selects the current data collected by a certain electric vehicle of Brilliance in the condition of New European Driving Cycle (NEDC) working condition as the battery model input. Then we get the power battery SOC, battery terminal voltage, and temperature and other internal parameters through the battery model. The battery model is shown in Figure 4.

The generated battery data is shown in Figure 5.

4.1. Analysis of Battery Parameter Identification Results Based on Improved GA Algorithm. We use the real current data under NEDC conditions and the terminal voltage data generated according to Figure 4 and preform the parameter identification of SOC in the range of $[0.3,0.9]$ under the PNGV battery model by the improved GA algorithm [25]. Then we observe the variation of the model parameters and compare it with the description of the corresponding parameter characteristics in the LG battery manual to verify the effectiveness of the improved GA algorithm in model parameter identification. The identification results are shown in Table 1.

The identification result shows that at the temperature of $26^{\circ} \mathrm{C}$, as the SOC of the power battery decreases, the ohmic internal resistance will increase, the polarization internal resistance gradually increases, and the battery capacitance gradually decreases. These changes are consistent with the change in the electrical characteristics of the battery. It can reflect that the improved GA algorithm proposed in this paper has certain effectiveness in model identification. 


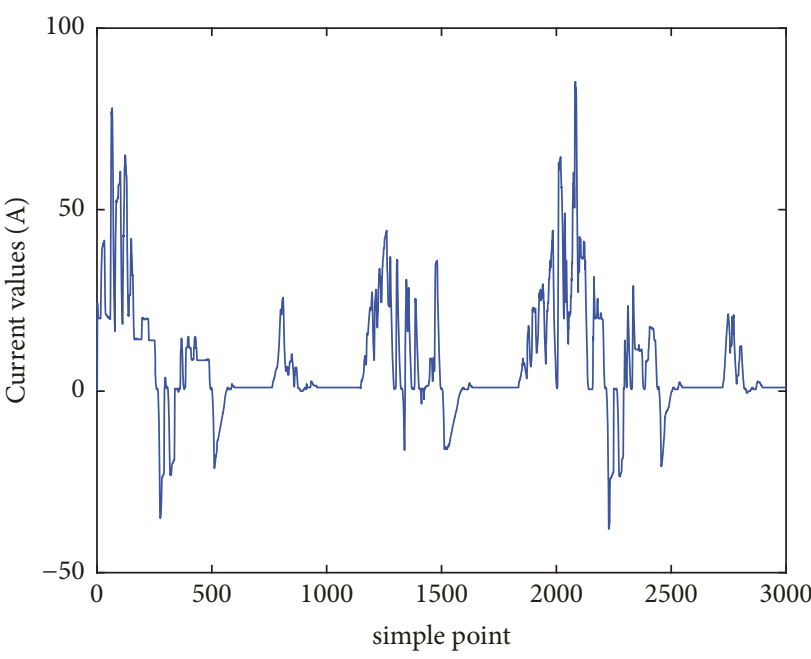

(a) The current data under NEDC condition

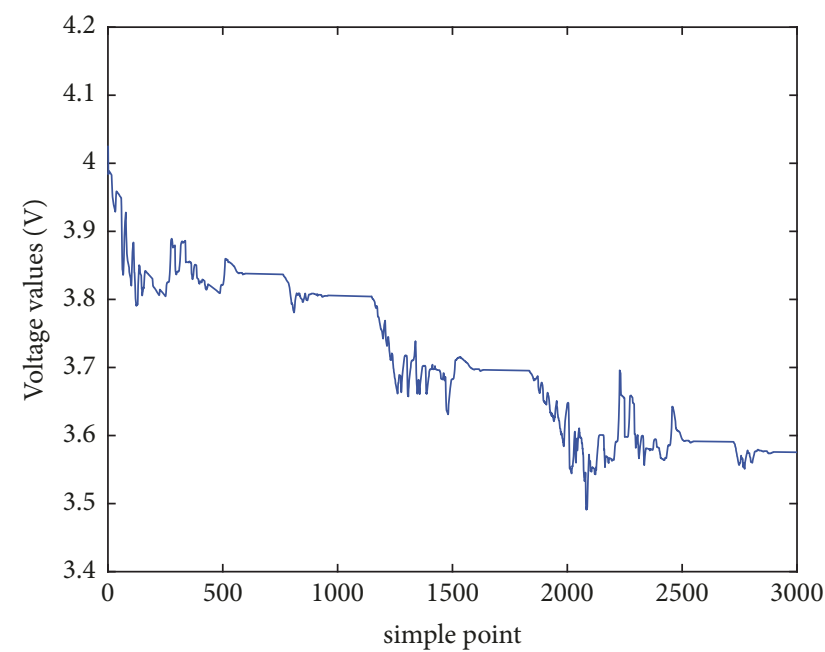

(b) The voltage data under NEDC condition

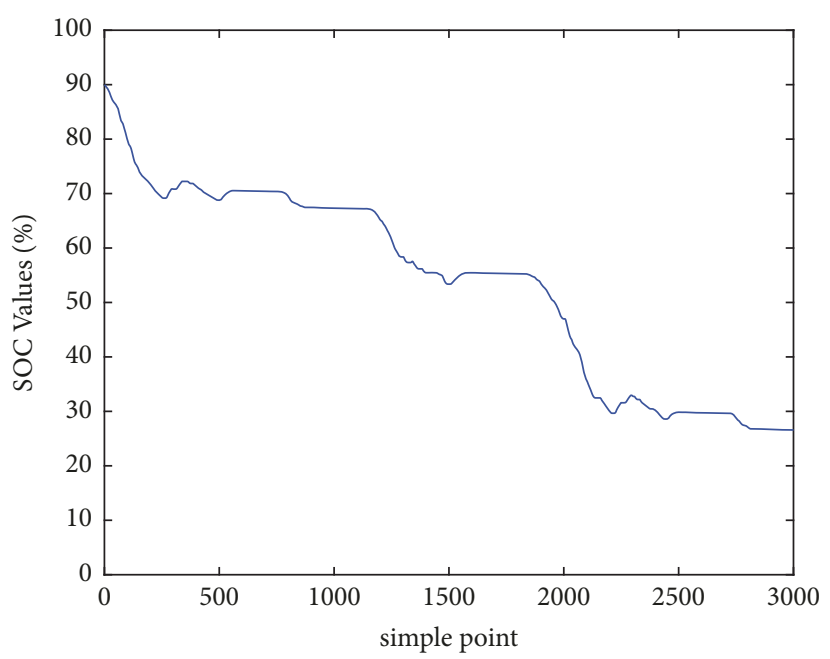

(c) SOC reference values

Figure 5: Battery data.

TABLE 1: Model parameter identification result.

\begin{tabular}{lccccc}
\hline SOC & $U_{o c v} / V$ & $C_{b} / F$ & $C_{p} / F$ & $R_{p} / m \Omega$ & 14.2 \\
\hline 0.3 & 3.5941 & 36857.4 & 15252.2 & 9.8 & 324.7 \\
0.4 & 3.6242 & 31950.3 & 12191.3 & 8.3 & 301.6 \\
0.5 & 3.6603 & 42604.9 & 25085.6 & 5.6 & 270.5 \\
0.6 & 47842.6 & 57508.2 & 4.8 & 165.3 \\
0.7 & 3.7302 & 53736.3 & 33678.4 & 4.45 & 78.6 \\
0.8 & 3.8350 & 57507.5 & 25563.7 & 1.6 & 10.1 \\
0.9 & 3.9260 & 58284.1 & 11203.4 & 2.4 \\
\hline
\end{tabular}

\subsection{Algorithm Verification Analysis under Noiseless Conditions}

4.2.1. Improved GA-UPF Algorithm Accuracy Verification. Firstly, in the noiseless environment, the improved GAUPF algorithm is used to estimate the SOC to verify its effectiveness. At the same time, we compare it with LS-UPF algorithm and improved GA-UKF algorithm. The verification results are shown in Figure 6.

The basic parameters: $N=50, n=16, \mathrm{G}=30, P_{\text {cor }}=0.8$, $P_{\text {mut }}=0.01, \alpha=0.5, k=2, \beta=0$.

In Figure 6(a), the solid line is the estimated result of the SOC by the improved GA-UPF algorithm. The black line is the estimation result of the SOC by the improved 


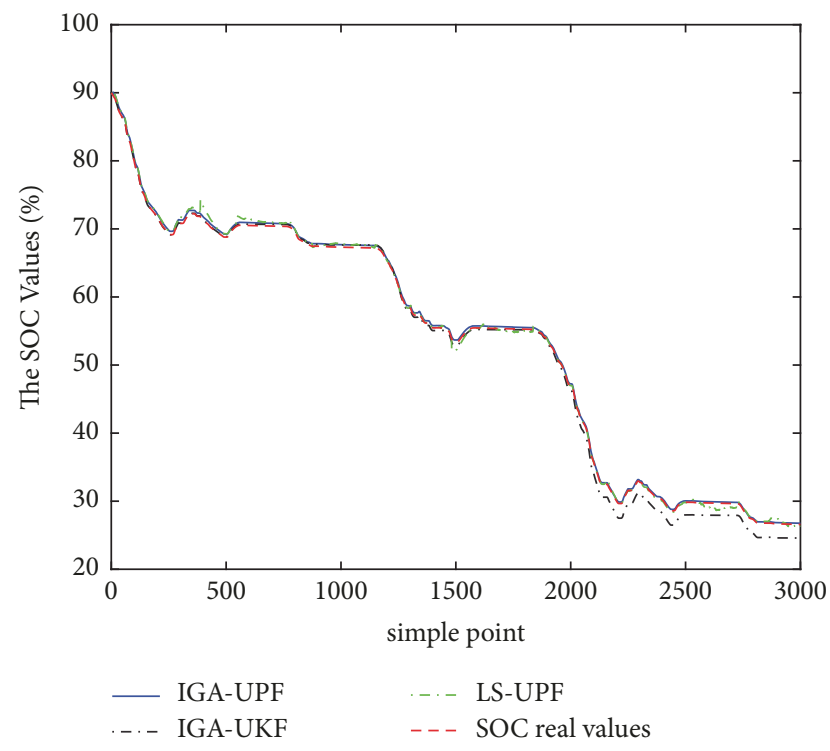

(a) SOC estimation results

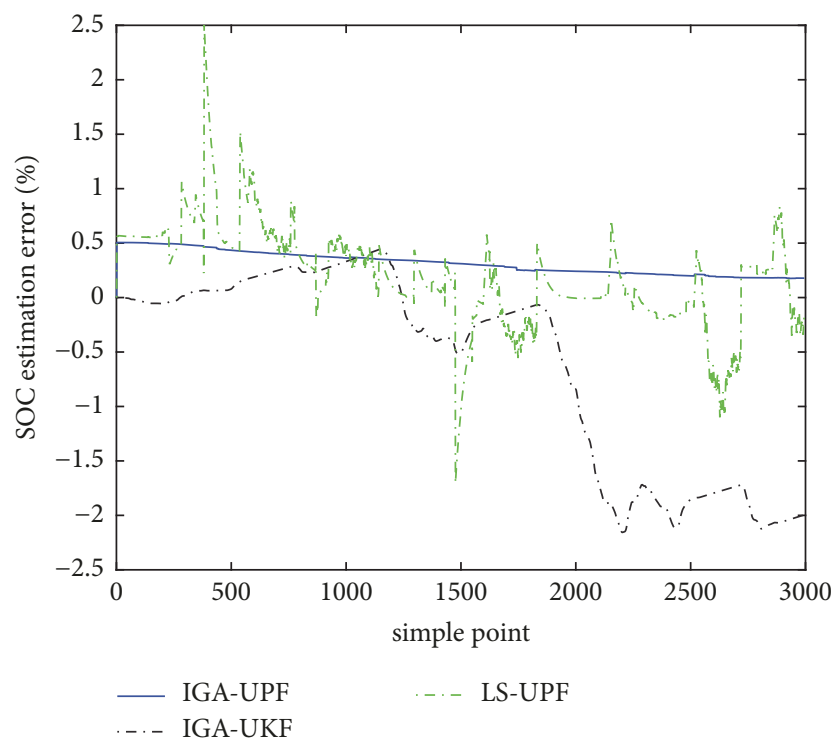

(b) SOC estimation error comparison curve

FIGURE 6: SOC estimation and error comparison results.

GA-UKF algorithm, the green line is the estimation result of the SOC by the LS-UPF algorithm, and the red line is the SOC reference values. Comparison result shows that the proposed SOC estimation method has higher estimation accuracy, and the estimation result is closer to the SOC reference value. In Figure 6(b), the solid line is the error value of the SOC estimation result based on the improved GA-UPF algorithm, and the result is convergent and relatively stable. The black line indicates the error of the SOC estimation result based on the improved GA-UKF algorithm, and the error fluctuation is larger than solid line. The green line indicates the error of the SOC estimation result based on the LS-UPF algorithm, and the error fluctuation is much larger than the other two algorithms. It reflects that for nonlinear time-varying systems such as power batteries, the accuracy of model parameter identification based on LS algorithm is poor, which directly affects the accuracy of SOC estimation. It also reflects the accuracy of the improved GA-UPF algorithm proposed in this paper.

4.2.2. Improved GA-UPF Algorithm Robustness Verification. In order to verify the robustness of the improved GA-UPF algorithm, the following experiment is performed: the initial SOC is set to $20 \%$ and $S O C_{\text {real }}=90 \%$. We use a relatively large error to simulate the strong interference factors such as outliers in the actual operating conditions of electric vehicles and verify the self-correcting robustness of the algorithm. The verification results are shown in Figure 7.

The result in Figure 7 shows that the improved GAUPF algorithm can converge to the vicinity of the real SOC within 10 iterations when there is a large deviation in the initial SOC. The error is $3 \%$ after 10 iterations and finally can converge to $1 \%$. It is proved that the improved GA-UPF algorithm proposed in this paper has great robustness in SOC estimation of power battery. It can converge to true value quickly under strong anomaly disturbance and has strong self-correction ability. Therefore, it is suitable for the SOC estimation problem of power battery with complicated and varied operating conditions.

\subsubsection{Improved GA-UPF Algorithm Stability Verification.} Considering that the traditional GA algorithm has a certain randomness problem, we propose the idea of using the LS algorithm to determine the initial search range. We carry out the following verification: under the same driving conditions, the LS-UPF algorithm, the improved GA-UKF algorithm, and the improved GA-UPF algorithm are used to perform multiple experiments on the same data sequence. The experimental results are shown in Table 2.

It can be seen from Table 2 that the improved GA-UPF algorithm proposed in this paper maintains a relatively stable absolute error and root mean square error in the process of multiple verifications and has good stability. In addition, compared with the SOC estimation method based on LS-UPF and improved GA-UKF algorithm, the improved GA-UPF algorithm always has smaller error; that is, the maximum absolute error is about $0.5 \%$ and the root mean square error is about $0.2 \%$. The maximum absolute error of the LS-UPF algorithm is about $2.5 \%$, and the root mean square error fluctuates around $0.5 \%$. The maximum absolute error of the LS-UPF algorithm is about $3.34 \%$, and the root mean square error fluctuates around $1.5 \%$. It is proved that the improved GA-UPF algorithm proposed in this paper has better stability and accuracy.

4.2.4. Improved GA-UPF Algorithm Time Complexity Anal$y$ sis. We know that both GA and UPF algorithm are the optimization, and SOC estimation is a process that needs to be done in real time. Therefore, the algorithm operation efficiency must be guaranteed. We use the least squares method 


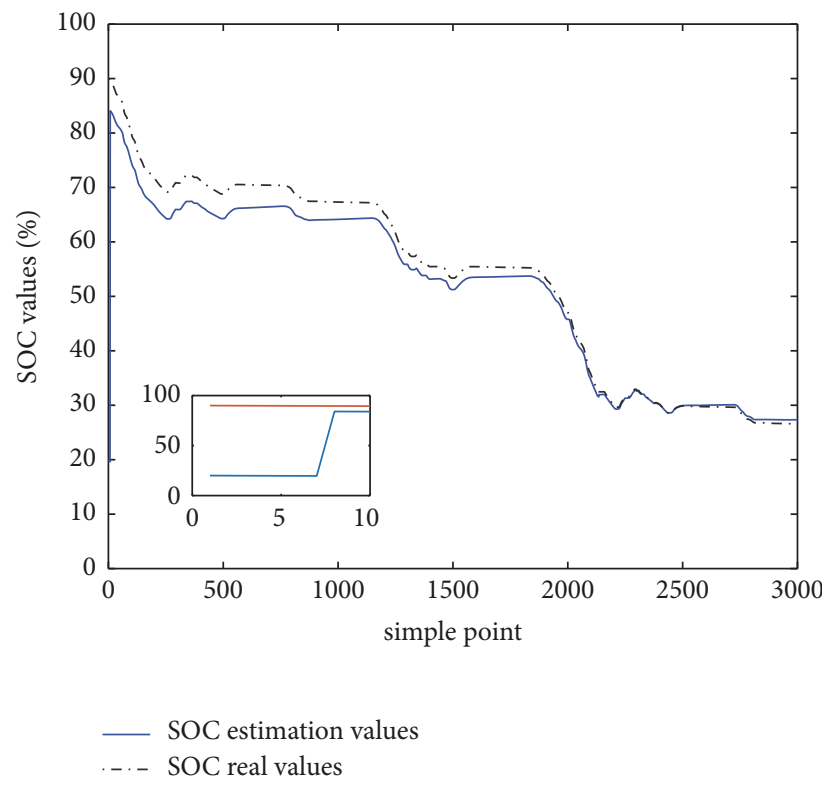

Figure 7: Comparison of SOC estimates.

TABLE 2: Improved GA-UPF algorithm stability verification results.

\begin{tabular}{lccr}
\hline Algorithm & Run times & MaxAE (\%) & RMSE(\%) \\
\hline LS-UPF & 70 & {$[2.39,2.51]$} & {$[0.5073,0.5386]$} \\
Improved GA-UKF & 70 & {$[2.16,3.34]$} & {$[1.1006,1.8569]$} \\
Improved GA-UPF & 70 & {$[0.44,0.51]$} & {$[0.2905,0.4125]$} \\
\hline
\end{tabular}

to reduce the initial search range of the GA algorithm. Then, the efficiency of the GA algorithm is improved. With the improved efficiency of the GA algorithm, the time complexity of the improved GA-UPF algorithm proposed in this paper is also reduced. We analyze the time complexity of improving the GA-UPF algorithm. The analysis results are shown in Table 3.

It can be seen from Table 3 that the improved GA-UPF algorithm has a single execution time of $46.3 \mathrm{~ms}$ and a total execution time of $138.9 \mathrm{~ms}$. The LS-UPF algorithm has a single execution time of $44.1 \mathrm{~ms}$ and the improved GAUKF algorithm has a single execution time of $41.7 \mathrm{~ms}$. By comparison, we can see that the improved GA-UPF algorithm has a $2.2 \mathrm{~ms}$ increase in single run time compared to the LSUPF algorithm and an increase of $4.6 \mathrm{~ms}$ over the single run time of the improved GA-UKF algorithm. The growth rate is $5 \%$ and $10 \%$, respectively. The extent of this increase is within our acceptance. Additionally, the current sensor on our electric car has a sampling period of $50 \mathrm{~ms}$. Therefore, the improved GA-UPF algorithm can meet engineering needs.

\subsection{Improved GA-UPF Algorithm Verification under Noisy Environment}

4.3.1. Algorithm Precision Verification in Gaussian Noise Environment. We know that in the actual driving conditions, the vehicle will inevitably encounter a variety of noise interference. If the algorithm is verified only in the noiseless environment, the noise immunity of the algorithm cannot be examined. Therefore, we verify the performance of the improved GA-UPF algorithm in a noisy environment. We add $30 \mathrm{~dB}$ of Gaussian white noise data to the current and $60 \mathrm{~dB}$ of Gaussian white noise to the voltage. The noise signal is shown in Figure 8. Then we use noise data to perform SOC estimation based on the improved GA-UPF algorithm. The estimated results are shown in Figure 9.

The basic parameters: $N=50, n=16, \mathrm{G}=30, P_{\text {cor }}=0.8$, $P_{\text {mut }}=0.01, \alpha=0.5, k=2, \beta=0$.

It can be shown in Figure 9 that the improved GA-UPF algorithm proposed in this paper still has good estimation accuracy, and the error is relatively stable. However, the accuracy of the improved GA-UKF algorithm drops to about $4.5 \%$. And the estimation accuracy of LS-UPF algorithm is seriously deviated; especially in 2000-2500 iterations, the SOC estimation error of the LS-UPF algorithm reaches $12 \%$. Such error is not allowed in engineering applications.

In order to verify the SOC estimation accuracy of the improved GA-UPF algorithm under different noise environments, we added $60 \mathrm{~dB}$ of Gaussian white noise to the current data and $80 \mathrm{~dB}$ of Gaussian white noise to the voltage data. The data with noise is shown in Figure 10. Then we use the data of Figure 10 to estimate the SOC. Then we compare it with the SOC estimation results obtained by the battery data of Figure 8. The comparison results are shown in Figure 11.

The result shows that the "solid line" SOC estimation results representing strong noise are basically consistent with 


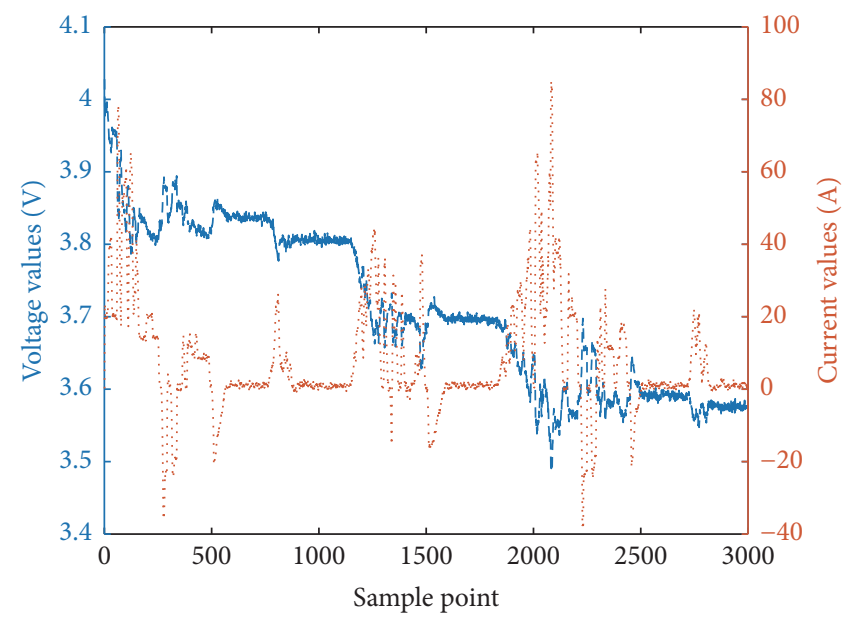

FIGURE 8: Current with $30 \mathrm{~dB}$ noise and voltage with $60 \mathrm{~dB}$ noise.

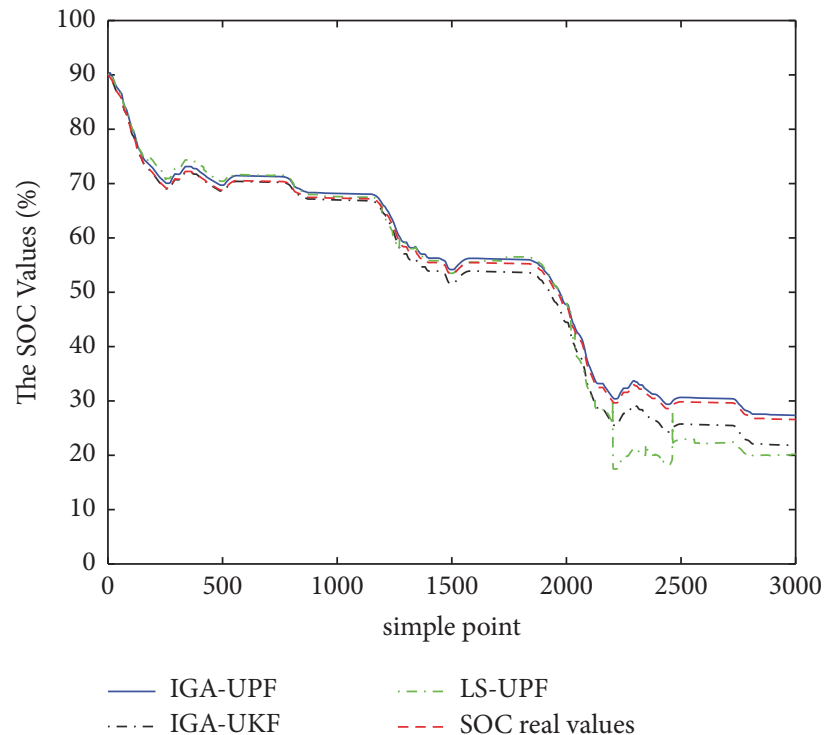

(a) SOC estimation result

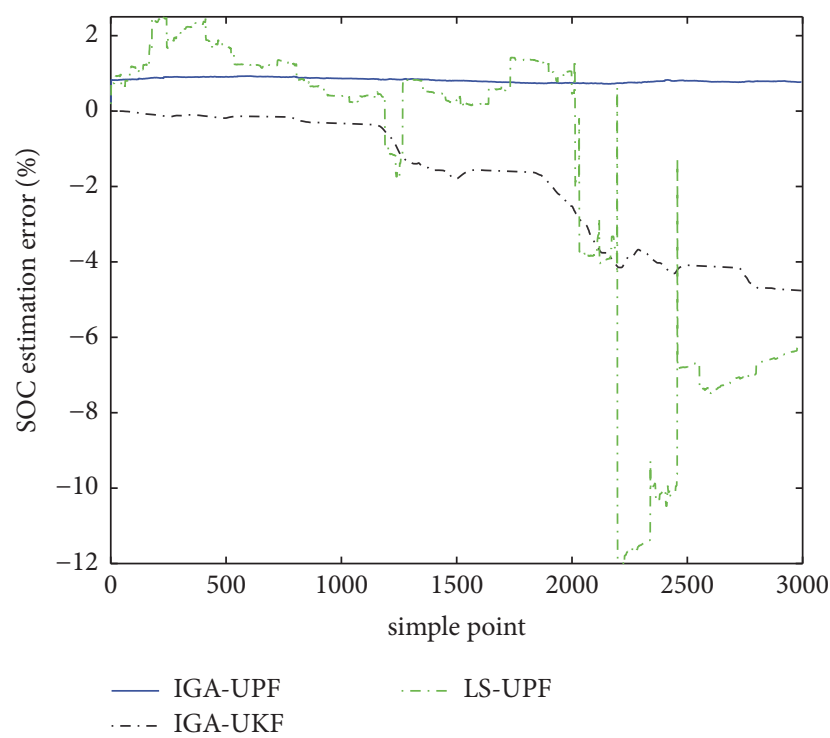

(b) SOC estimation error curve

FIGURE 9: Verification results in noisy environments.

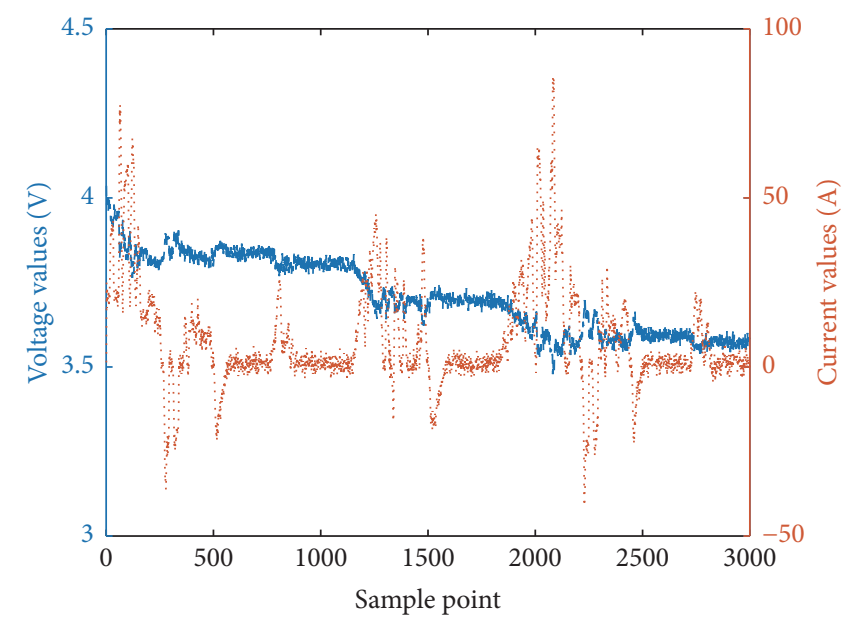

FIGURE 10: Current with $60 \mathrm{~dB}$ noise and voltage with $80 \mathrm{~dB}$ noise. 
TABLE 3: Improved GA-UPF algorithm time complexity analysis results.

\begin{tabular}{lccc}
\hline Algorithm & Running Times & Average Time for One Iteration & Total Running Time \\
\hline LS-UPF & 70 & $44.1 \mathrm{~ms}$ & $132.3 \mathrm{~s}$ \\
Improved GA-UKF & 70 & $41.7 \mathrm{~ms}$ & $125.1 \mathrm{~s}$ \\
Improved GA-UPF & 70 & $46.3 \mathrm{~ms}$ & $138.9 \mathrm{~s}$ \\
\hline
\end{tabular}

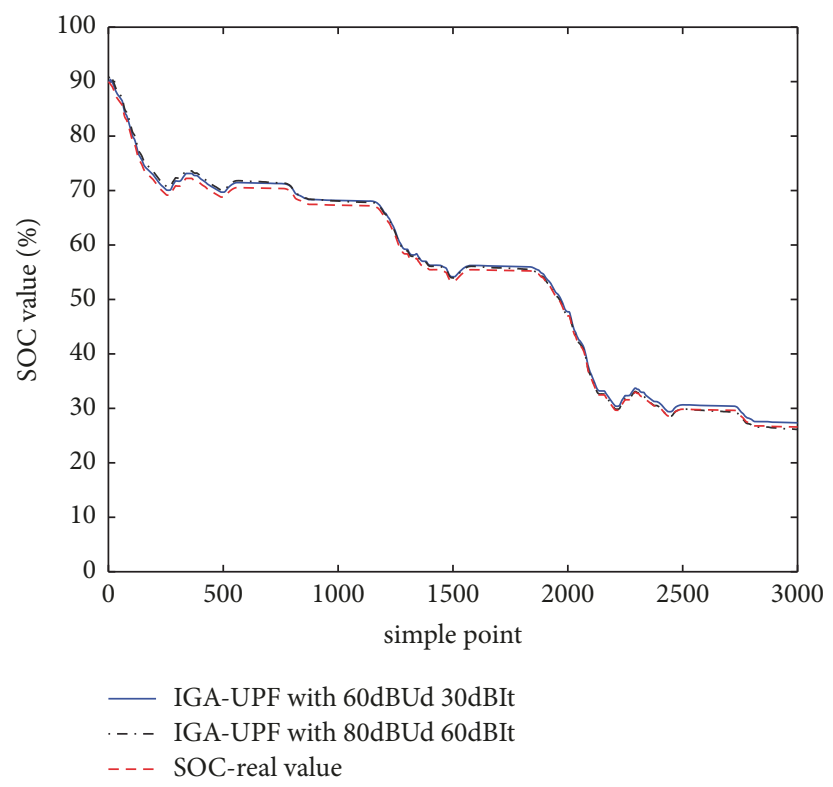

FIGURE 11: Comparison of SOC estimation results under different noise environments.

the "dotted line" SOC estimation results representing weak noise, and there is no significant accuracy deviation due to the difference in noise intensity. Therefore, it can be proved that the proposed SOC estimation method based on the improved GA-UPF algorithm has strong antinoise ability. Furthermore, it can be reflected from one side that the improved GA algorithm proposed in this paper has good antinoise ability and can accurately identify the parameters of PNGV model under noisy environment. It effectively reduces the adverse effects of noise on SOC estimation in engineering applications.

4.3.2. Algorithmic Accuracy Verification in Non-Gaussian Noise Environment. Considering the complex and variable noise environment of electric vehicles in engineering applications, we verify the SOC estimation performance of the improved GA-UPF algorithm in the colored noise environment. The data for adding colored noise is shown in Figure 12. The SOC estimation results are shown in Figure 13.

The result shows that the improved GA-UPF algorithm also has good SOC estimation accuracy under colored noise. The maximum absolute error of the SOC estimation is $0.7 \%$, and the convergence efficiency is excellent. It can be proved that the improved GA-UPF algorithm proposed in this paper also has strong antinoise ability in the colored noise environment. It is suitable for engineering applications in electric vehicles with complex noise environments. It also reflects the antinoise ability of the improved GA algorithm in parameter identification.

4.3.3. Improved GA-UPF Algorithm Stability Verification under Noisy Environment. In the environment of Gaussian white noise and colored noise, the stability of the estimation accuracy of the improved GA-UPF algorithm is verified. The result is shown in Table 4.

The result shows that under different white noise intensities, the SOC estimation based on the improved GA-UPF algorithm shows good stability under multiple verifications, and the maximum absolute error is less than $2.5 \%$, and the root mean square error is about $0.6 \%$. In the colored noise environment, the algorithm also shows good stability, the maximum absolute error is about $2 \%$, and the root mean square error is about $0.9 \%$. It is proved that the improved GAUPF algorithm proposed in this paper still has good stability under complex noise environment, is suitable for engineering applications, and has certain practicability. However, it can also be seen that the SOC estimation error will increase with the increase of the data noise intensity. It is also explained from one side that the noise reduction process is necessary for any algorithm when the noise intensity is too high.

\section{Conclusions}

In this paper, an online identification method for power battery model parameters based on improved GA algorithm 


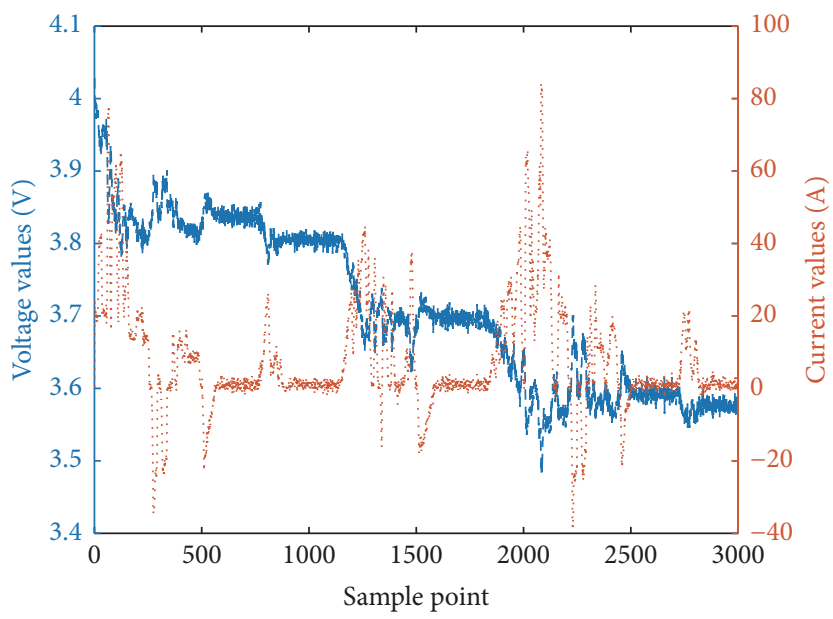

FIgURE 12: Data with colored noise.

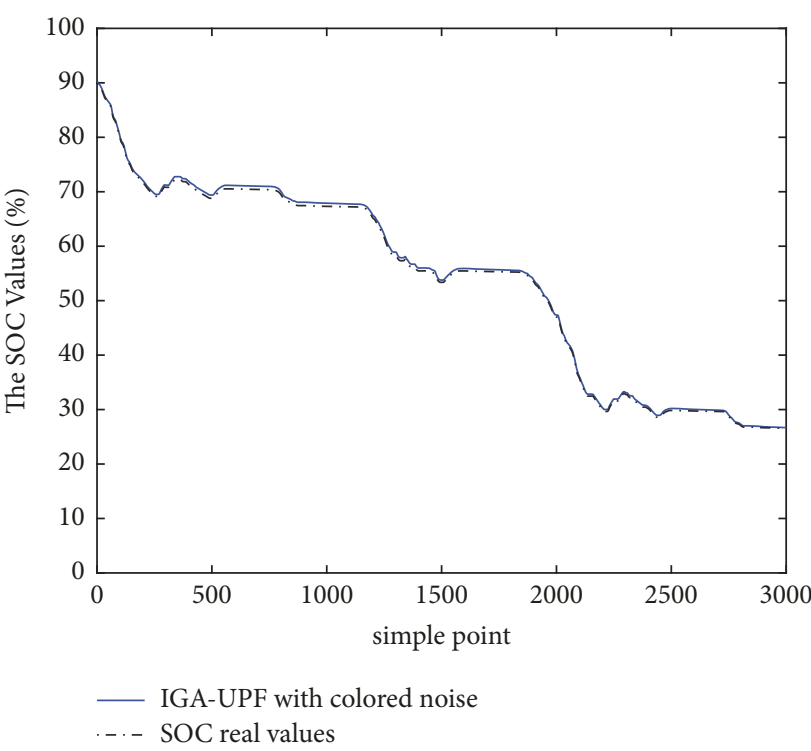

(a) SOC estimation results in a colored noise environment

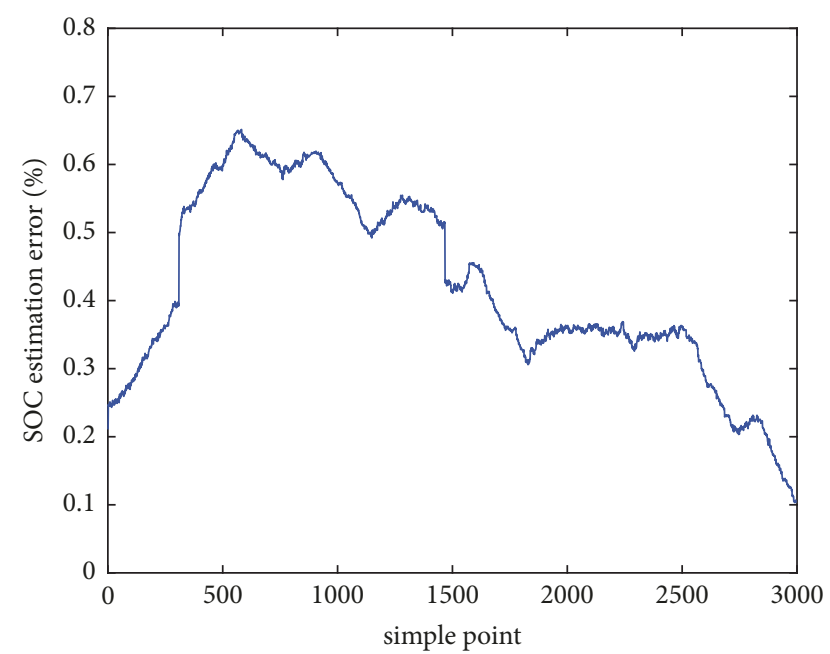

IGA-UPF with colored noise

(b) SOC estimation error under colored noise

FIGURE 13: SOC estimation results under colored noise.

is proposed to solve the problem that SOC estimation accuracy is highly dependent on model parameters. In addition, the driving conditions of electric vehicles are complex and variable, which makes the collected current signals have a lot of noise, abnormal interference signals, and spike transient signals. Therefore, this paper proposes a power battery SOC estimation method that combines improved GA algorithm with UPF. The advantages of this algorithm are as follows.

(1) The improved GA algorithm is more suitable for online identification of power battery parameters with nonlinear and complex time-varying characteristics. It can converge quickly and reduce the probability of falling into local optimum. It can meet engineering requirements and improve the accuracy of model parameter identification.

(2) The improved GA algorithm combined with the $\mathrm{UPF}$ algorithm is used for SOC online estimation of electric vehicle power battery, which has strong robustness, can effectively suppress various types of noise and outlier interference, and makes the algorithm have better stability.

In the simulation and verification section, the stability, noise immunity, robustness, and estimation accuracy of the SOC estimation method based on the improved GAUPF algorithm are verified by comparison with the LS-UPF algorithm and improved GA-UKF algorithm. The simulation results demonstrate the effectiveness and practicability of the improved GA-UPF algorithm.

\section{Data Availability}

The data used in this paper comes from the experimental data of the OEM. It is currently a trade secret and should not be disclosed. 
TABLE 4: Improved GA-UPF algorithm stability verification under noisy environment.

\begin{tabular}{lcccc}
\hline Algorithm & noise & Run times & MaxAE(\%) & RMSE(\%) \\
\hline Improved GA-UPF & $60 d B U_{d} ; 30 d B I_{t}$ & 70 & {$[0.5042,1.4236]$} & {$[0.3689,0.6626]$} \\
Improved GA-UPF & $80 d B U_{d} ; 60 d B I_{t}$ & 70 & {$[1.6503,2.2333]$} & {$[0.8029,0.9867]$} \\
Improved GA-UPF & Colored noise & 70 & {$[0.4502,1.6896]$} & {$[0.3147,0.9718]$} \\
\hline
\end{tabular}

\section{Conflicts of Interest}

The authors declare that they have no conflicts of interest.

\section{Acknowledgments}

This work was supported in part by Tianjin Province Science and Technology projects (Grant no. 18JCQNJC77200) and National key research and development plan project (Grant no. 2017YFB1103003, 2016YFB1100501).

\section{References}

[1] R. Zhang, B. Xia, B. Li et al., "State of the art of lithium-ion battery SOC estimation for electrical vehicles," Energies, vol. 11, no. 7, p. 1820, 2018.

[2] M. A. Hannan, M. S. H. Lipu, A. Hussain, and A. Mohamed, "A review of lithium-ion battery state of charge estimation and management system in electric vehicle applications: Challenges and recommendations," Renewable \& Sustainable Energy Reviews, vol. 78, pp. 834-854, 2017.

[3] R. Xiong, J. Cao, Q. Yu, H. He, and F. Sun, "Critical review on the battery state of charge estimation methods for electric vehicles," IEEE Access, vol. 6, pp. 1832-1843, 2017.

[4] Z. Li, J. Huang, B. Y. Liaw, and J. Zhang, "On state-of-charge determination for lithium-ion batteries," Journal of Power Sources, vol. 348, pp. 281-301, 2017.

[5] H. Yu, R. Lu, C. Zhu, and R. Ma, "State of charge estimation calibration for Ni-MH battery based on Ampere-hour method," Diangong Jishu Xuebao/Transactions of China Electrotechnical Society, vol. 27, no. 6, pp. 12-18, 2012.

[6] Z.-H. Chen, L. Zhong, Y. He, and C.-B. Zhang, "Method to calibrate and estimate Li-ion battery state of charge based on charging method," Kongzhi yu Juece/Control and Decision, vol. 29, no. 6, pp. 1148-1152, 2014.

[7] Z. Hongwei, L. Jiancheng, and Z. Bingbing, "SoC estimation of lithium-ion battery based on algorithm of $\mathrm{AH}$ with $\mathrm{PF}$ correction," Transducer and Microsystem Technologies, vol. 35, no. 10, pp. 4-7, 2016.

[8] S. Piller, M. Perrin, and A. Jossen, "Methods for state-of-charge determination and their applications," Journal of Power Sources, vol. 96, no. 1, pp. 113-120, 2001.

[9] Y. He, X. Liu, C. Zhang, and Z. Chen, "A new model for Stateof-Charge (SOC) estimation for high-power Li-ion batteries," Applied Energy, vol. 101, pp. 808-814, 2013.

[10] R. Xiong, F. Sun, Z. Chen, and H. He, "A data-driven multi-scale extended Kalman filtering based parameter and state estimation approach of lithium-ion olymer battery in electric vehicles," Applied Energy, vol. 113, pp. 463-476, 2014.

[11] M. Ye, H. Guo, and B. Cao, "A model-based adaptive state of charge estimator for a lithium-ion battery using an improved adaptive particle filter," Applied Energy, vol. 190, pp. 740-748, 2017.
[12] L.-L. Li, Y.-H. Ren, C.-H. Wang, and C.-T. Jen, "A new method to estimate the state of charge of the green battery," Microelectronics Reliability, vol. 79, pp. 306-313, 2017.

[13] B. Xia, Z. Lao, R. Zhang et al., "Online parameter identification and state of charge estimation of lithium-ion batteries based on forgetting factor recursive least squares and nonlinear Kalman filter," Energy, vol. 11, no. 1, pp. 1-23, 2018.

[14] G. L. Plett, "Extended Kalman filtering for battery management systems of LiPB-based HEV battery packs-part 2. Modeling and identification," Journal of Power Sources, vol. 134, no. 2, pp. 262-276, 2004.

[15] G. L. Plett, "Extended Kalman filtering for battery management systems of LiPB-based HEV battery packs. Part 3. State and parameter estimation," Journal of Power Sources, vol. 134, no. 2, pp. 262-276, 2004.

[16] F. Sun, X. Hu, Y. Zou, and S. Li, "Adaptive unscented Kalman filtering for state of charge estimation of a lithium-ion battery for electric vehicles," Energy, vol. 36, no. 5, pp. 3531-3540, 2011.

[17] W. Wang, X. Wang, C. Xiang, C. Wei, and Y. Zhao, "Unscented kalman filter-based battery SOC estimation and peak power prediction method for power distribution of hybrid electric vehicles," IEEE Access, vol. 6, pp. 35957-35965, 2018.

[18] G. L. Plett, "Sigma-point Kalman filtering for battery management systems of LiPB-based HEV battery packs. Part 2: Simultaneous state and parameter estimation," Journal of Power Sources, vol. 161, no. 2, pp. 1369-1384, 2006.

[19] E. Chemali, P. J. Kollmeyer, M. Preindl, R. Ahmed, and A. Emadi, "Long short-term memory-networks for accurate state of charge estimation of Li-ion batteries," IEEE Transactions on Industrial Electronics, vol. 65, no. 8, pp. 6730-6739, 2018.

[20] Y. Ma, P. Duan, Y. Sun, and H. Chen, "Equalization of lithiumion battery pack based on fuzzy logic control in electric vehicle," IEEE Transactions on Industrial Electronics, vol. 65, no. 8, pp. 6762-6771, 2018.

[21] C. Song, Y. Shao, S. Song et al., "Energy management of parallelconnected cells in electric vehicles based on fuzzy logic control," Energies, vol. 10, no. 3, p. 404, 2017.

[22] J. Meng, G. Luo, and F. Gao, "Lithium polymer battery stateof-charge estimation based on adaptive unscented kalman filter and support vector machine," IEEE Transactions on Power Electronics, vol. 31, no. 3, pp. 2226-2238, 2016.

[23] X. Liu, W. Li, and A. Zhou, "PNGV equivalent circuit model and soc estimation algorithm for lithium battery pack adopted in AGV vehicle," IEEE Access, vol. 6, pp. 23639-23647, 2018.

[24] Y. Guo, Z. Zhao, and L. Huang, "SoC estimation of lithium battery based on AEKF algorithm," Energy Procedia, vol. 105, pp. 4146-4152, 2017.

[25] W. Gao, M. Jiang, and Y. Hou, "Research on PNGV model parameter identification of LiFePO4 Li-ion battery based on FMRLS," in Proceedings of the 2011 6th IEEE Conference on Industrial Electronics and Applications, ICIEA 2011, pp. 22942297, China, June 2011. 
[26] L. Wang, D. Lu, Q. Liu, L. Liu, and X. Zhao, "State of charge estimation for LiFePO4 battery via dual extended kalman filter and charging voltage curve," Electrochimica Acta, vol. 296, pp. 1009-1017, 2019.

[27] D. Liu, X. Yin, Y. Song, W. Liu, and Y. Peng, "An on-line state of health estimation of lithium-ion battery using unscented particle filter," IEEE Access, vol. 6, pp. 40990-41001, 2018.

[28] L. R. Haupt, "An introduction to genetic algorithms for electromagnetics," IEEE Antennas Propagation Magazine, vol. 37, no. 2, pp. 7-15, 2002. 


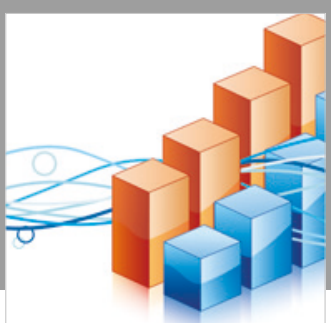

Advances in

Operations Research

\section{-n-m}
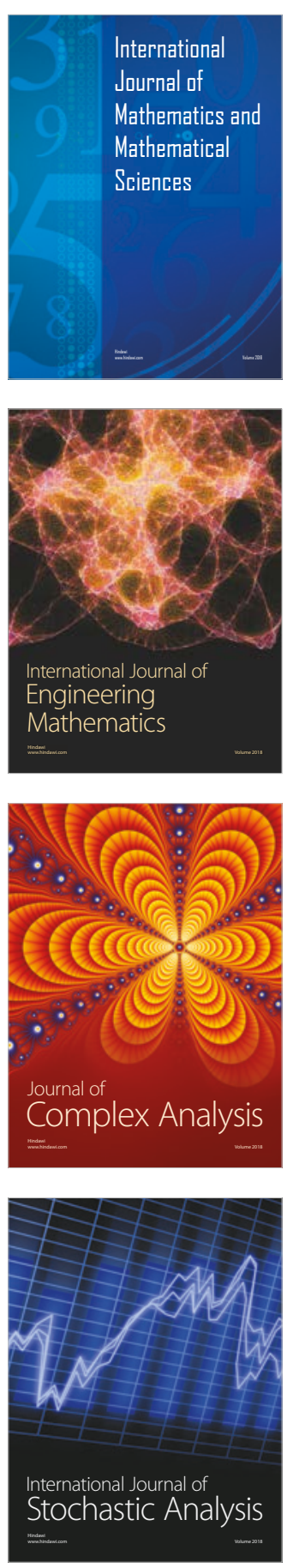
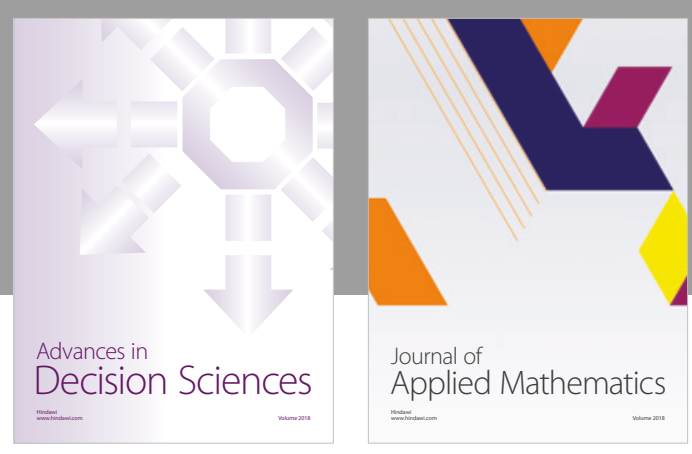

Journal of

Applied Mathematics
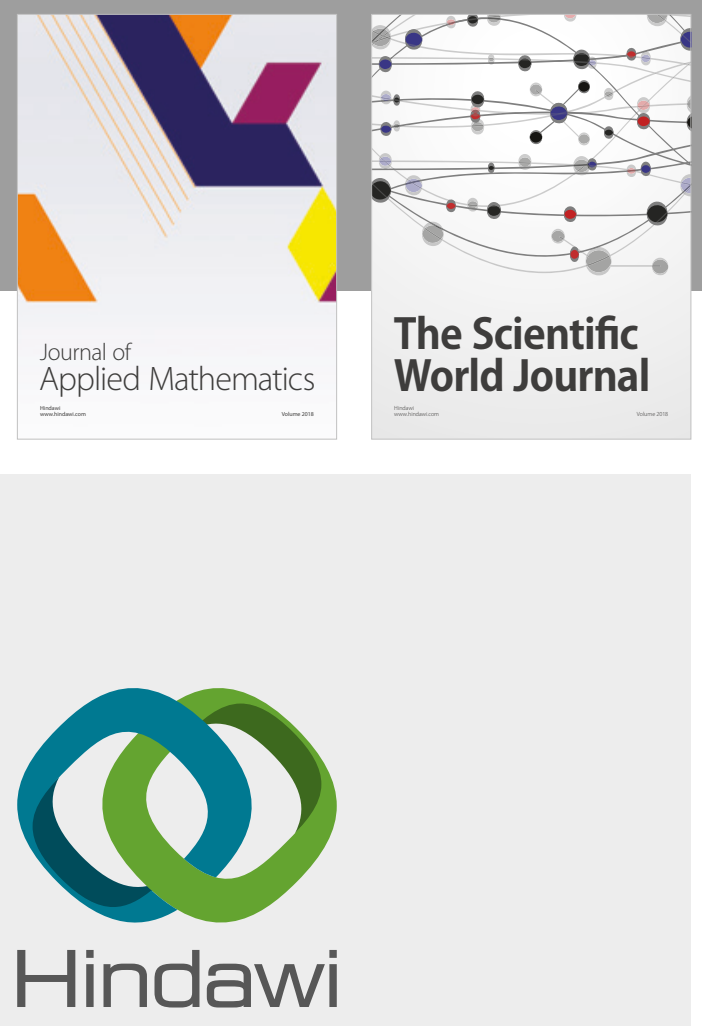

Submit your manuscripts at

www.hindawi.com

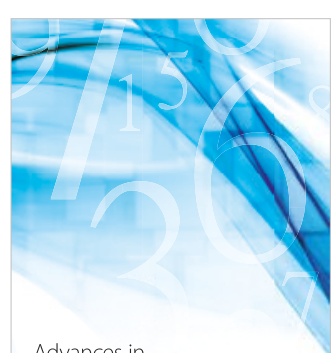

Advances in
Numerical Analysis
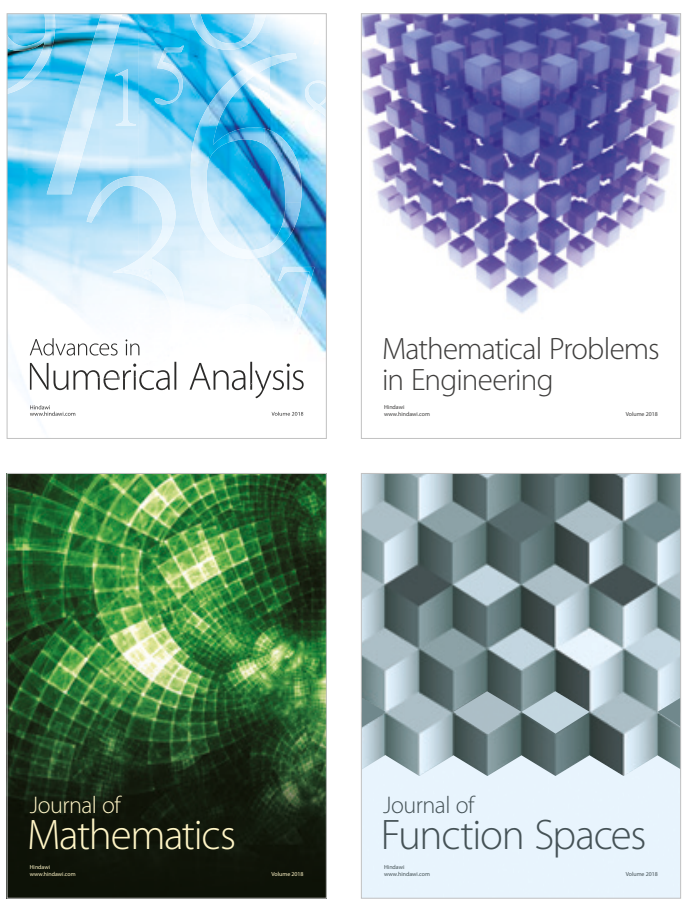

Mathematical Problems in Engineering

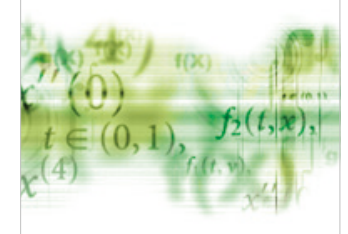

International Journal of

Differential Equations

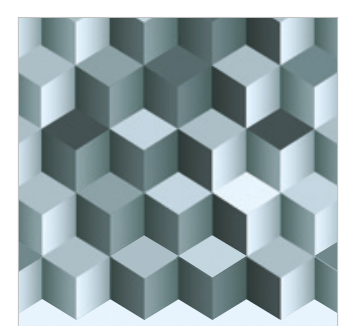

Journal of

Function Spaces

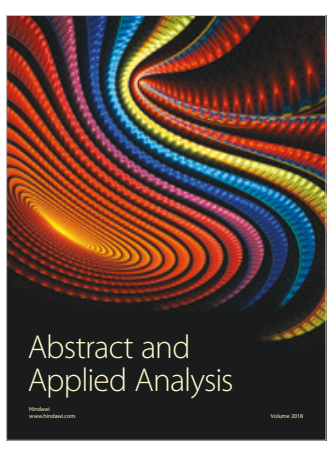

The Scientific

World Journal

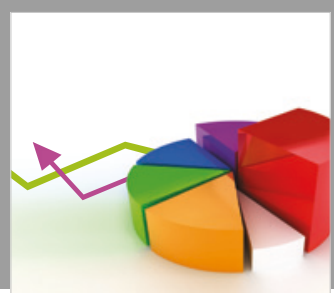

Journal of

Probability and Statistics
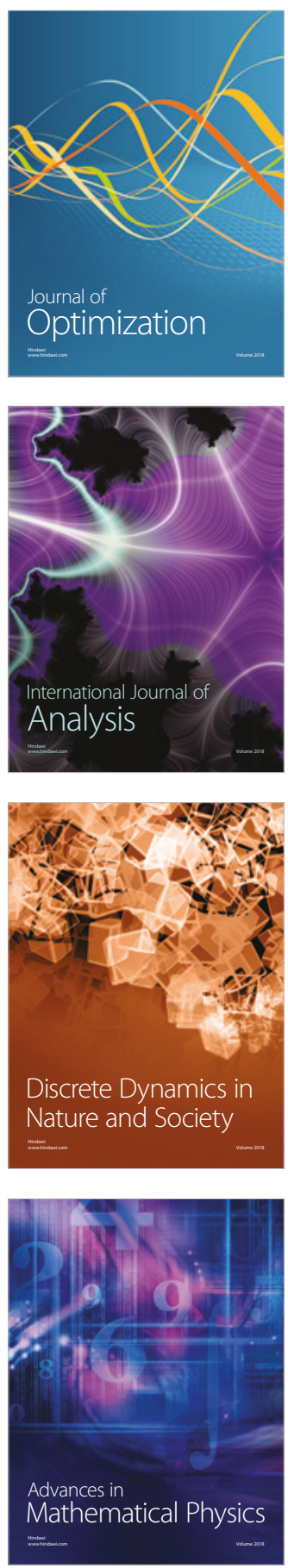